ORGINAL ARTICLE

\title{
The effect of Platelet Rich Plasma injection in treatment of Androgenetic alopecia patients
}

\author{
Ahmed Mohamed Kamal ELGarf ${ }^{1}$, Waleed Mohammad Al Balat ${ }^{1}$, Shimaa ElAraby Shaban \\ Othman $^{2 *}$ \\ ${ }^{1}$ Department of Dermatology, Venereology and Andrology, Zagazig University, Faculty of \\ Medicine \\ ${ }^{2}$ Department of Dermatology and Andrology, Zagazig General hospital
}

\author{
*Corresponding author: \\ Name: Shimaa ElAraby \\ Shaban, \\ Dermatologist at zagzig \\ General hospital, \\ mobile : 01027506829 \\ E-mail: \\ shaimaa1989a@gmail.com
}

$\begin{array}{ll}\text { Submit Date } & \text { 2019-07-07 } \\ \text { Revise Date } & \mathbf{2 0 1 9 - 0 8 - 2 4} \\ \text { Accept Date } & \mathbf{2 0 1 9 - 0 9 - 0 3}\end{array}$

\section{ABSTRACT}

Background: Androgenetic alopecia (AGA) is an androgen-dependent, hereditary dermatological disorder , affecting men and women causing progressive thinning of the scalp hair in a defined Characteristic pattern, with significant negative impact on social and psychological state of patients. Aim of the work: To evaluate the efficacy and safety of Platelet Rich Plasma injection in androgenetic alopecia patients. Subjects and methods: A therapeutic trial study was carried out on a sample size of 20 patients with AGA who attended the outpatients' clinics of dermatology department - Zagazig University Hospitals. Results: At baseline, 16 patients $(80 \%)$ had a positive pull test with a mean number of 9 hairs. At $4^{\text {th }}$ and $8^{\text {th }}$ week ,The hair loss decreased with a mean number of 5.5 and 3 hairs respectively. After PRP injection 2 patients (10\%) achieved high improvement , 6 patients $(30 \%)$ achieved moderate improvement , 6 patients $(30 \%)$ achieved mild improvement and 6 patients (30\%) achieved no change, with final patient's satisfaction (40\%). Conclusion: PRP injection is relatively effective in treatment of Androgenetic alopecia.

Key words: Platelet rich plasma; hereditary ; androgenetic alopecia

\section{INTRODUCTION}

A ndrogenetic Alopecia (AGA) is one of the most common non cicatritial patterned alopecia occuring in both male and female .It is a progressive thinning of the scalp hair causing considerable lowering of the self-confidence and psychological wellbeing of the patient. It is an androgen dependent dermatological disorder modulated through metabolite of testosterone dihydrotestosterone (DHT) and hair follicle related androgen receptor (AR). Some genetic factors also have been involved in its etiology [1] .

Treatment options proposed for promoting hair growth in androgenetic alopecia include topical Minoxidil , 5-alpha reductase inhibitors (finasteride and dutasteride), Anti-Androgens, Prostaglandin analogues, PRP and hair transplant surgery [2] .
Platelet-Rich Plasma (PRP) is an autologous preparation of human platelet in concentrated plasma . This concentrate enriched with various growth factors, cytokines and bioactive molecules, which are included in the regulation of hair morphogenesis and hair cycle ; stimulating the development of new follicles, increasing vascularization, and prolongation of the anagen phase [3].

\section{AIM OF THE WORK}

Aim of the work is to evalute the efficacy and safety of PRP injection in treatment of androgenetic alopecia .

\section{PATIENTS AND METHODS}

The work has been carried out in accordance with The Code of Ethics of the World Medical Association (Declaration of Helsinki) for studies involving humans.

Setting: 
This study was carried out in the Dermatology and Venereology DepartmentFaculty of Medicine-Zagazig University Hospitals, in the period from march 2018 till march 2019.

\section{Patients:}

Twenty males and females patients of different age, duration and grades of AGA were included in the study. The diagnosis of AGA was clinically proven by the characteristic pattern distribution of frontal and vertex hair in males according to Hamilton scale and the christmas tree pattern of diffuse hair loss at middle hairline in females according to Ludwig grade. Informed consent was taken from every patient and the study was approved by Institutional Review Board - (IRB) at Zagazig University.

\section{Inclusion criteria:}

- Age: 18-50 years.

- Normal platelet count ( 150,000 to 450,000 platelets per microliter of blood ).

\section{Exclusion criteria :}

-Pregnant female.

-Local infection of the scalp

-Patients who received any other treatment of AGA in the last six months

-Platelet dysfunction syndrome, uncontrolled DM, haemodynamic instability, sepsis, acute and chronic infections, chronic liver disease.

-Anticoagulant therapy (Aspirin, Heparin or Warfarin).

\section{All patients were subjected to:}

-Full history taking .

- Clinical examination (local and general).

-Investigations including complete blood picture (CBC), prothrombin time (PT),

and partial thromboplastin time (PTT).

\section{Method:}

Twenty patients ( 8 males and 12 females) aged $26-43$ years were treated with six sessions of PRP injections .

\section{Schedule:}

- Once every two weeks for three months, (week $0,2,4,6,8,10$ ).

\section{Preparation of PRP:}

To prepare PRP, double spin method had been used . $10 \mathrm{~mL}$ of each patient's blood was drawn manually using a 10-ml sterile injector containing $1 \mathrm{ml}$ of trisodium citrate as an anticoagulant then centrifuged by two centrifugation steps ; The first centrifugation step at $250 \mathrm{~g}$ for $10 \mathrm{~min}$ at room temperature and the second centrifugation step at $2000 \mathrm{~g}$ for $10 \mathrm{~min}$. The extracted PRP was transferred into $1 \mathrm{~mL}$ insulin syringes and activated by adding 0.1 $\mathrm{mL}$ of calcium chloride to $0.9 \mathrm{~mL}$ of PRP and made ready for injections [4].

\section{Technique of injection:}

Injection connected intradermally utilizing $1 \mathrm{ml}$ syringe and 30 guage sterile needle. Anatomical infusion locales on the scalp: frontal, parietal and vertex territories. Before injection, the objective scalp surface was cleaned with alcohol pads. Approximately $0.1 \mathrm{ml}$ is injected per site at a $1 \mathrm{~cm}$ interval, at a depth of $2-4 \mathrm{~mm}$ and at an angle of 30-60.The total volume injected each session ranged from $1 \mathrm{ml}$ to $2 \mathrm{ml}$ according to size of the injected area.

\section{Evaluation : \\ - Hair pull test:}

The hair pull test was performed ; At the first session, 4th week, 8th week ,and at the end of treatment. All patients were refrained from washing hair two days before the test. A bundle of about 50-60 hairs were grasped between the thumb, index and middle finger from the base close to the scalp. The hair was firmly pulled away from the scalp and the removed hair was counted .

\section{- Physician and photographic assessments:}

Independent observer assessment of photographs was done by comparing standardized digital photographs taken before and after the treatment and The degree of improvement evaluated using a seven-point scale as follows ; great improved (score 3), moderate improved (score 2), mild improved (score 1), unchanged (score 0), mild aggravated (score -1), moderate aggravated (score -2), and great aggravated (score -3) [5].

\section{- Patient satisfaction:}

A questionnaire was given to patients at the end of treatment to assess patient satisfaction as Satisfied or Not satisfied.

\section{- Statistical Analysis:}

All statistical analysis were performed using IBM SPSS Statistics, version 24 (IBM; Armonk, New York, USA). Continuous variables were presented by the mean $\pm \mathrm{SD}$ if normally distributed or median(range) if not 
normally distributed. Categorical variables were presented by the count and percentage if nominal or median(range) if ordinal, The following tests were used; Friedman test , Fisher Exact Test and Dunn's post hoc test.

\section{RESULTS}

This study was carried out on twenty patients with AGA . Classification of males according to Norwad Hamilton scale showed that 2 patients were of type II AGA, 4 patients were of type IIIv and 2 patients was of type IV,. Using Ludwig's classification in females, 10 patients were of type and 2 patients was of type AGA.. Family history was +ve in 12 patients and - ve in 8 patients . 6 patients received minoxdil, and 14 patients received no therapy. The disease duration ranged between 2-12 years.

\section{Therapeutic response:}

The response to treatment was assessed using: Hair pull test , physician assessment, photographic assessment and patient satisfaction.

\section{- Hair pull test assessment:}

Before treatment, 16 patients $(80 \%)$ had a positive pull test with a mean number of 9 hairs. At 4th and 8th week, The hair loss decreased with a mean number of 5.5 and 3 hairs respectively. The hair loss was continued to decrease and all patient (100\%) was negative with a mean number of 2 hairs at the end of treatment this is highly statistically significant $(\mathrm{P}<.001)$, ( figure 1 ).

Dunn's post hoc test revealed that median hair loss highly significantly decreased at 8th week and at the end PRPsessions compared to before initiation of PRP therapy $(P=.008$ vs $P<.001)$, but the other differences were not statistically significant $(P>.05)$, as shown in (Table 1$)$.

- Physician and photographic assessments:

Independent observer assessment of photographs, revealed that $(30 \%)$ perceived as no change (score $=0), \quad 30 \%$ as mild improvement (score $=1$ ), $30 \%$ as moderate improvement (score $=2$ ) and $10 \%$ as high improvement (score $=3$ ) no statistically significant difference was detected between both sexes .(figure 2 and 3 ).

\section{- Regarding patient satisfaction:}

12 patients $(60 \%)$ were not satisfied and 8 patients $(40 \%)$ were satisfied with no serious or persistent side effects were detected during the study.Only mild and transient adverse events were observed and resolved the next day without treatment as follows; $60 \%$ experienced no side effects, $40 \%$ of patients experienced mild headache .

Table 1: Dunn's post hoc test for hair pull test of PRP injection showing number of hair pulled ( before treatment sessions, at 4th week, at 8th week and at the end of the treatment).

\begin{tabular}{|l|l|l|l|l|}
\hline Hair pull test & $\begin{array}{l}\text { Before } \\
\text { treatment } \\
\text { sessions }\end{array}$ & At $\mathbf{4}^{\text {th }}$ week & At $\mathbf{8}^{\text {th }}$ week & $\begin{array}{l}\text { At the end of } \\
\text { the treatment }\end{array}$ \\
\hline$n=10$ & $n=10$ & $n=10$ & $n=10$ \\
\hline median(range) & $9(3-13)$ & $5.5(3-10)$ & $3(2-9)$ & $3(1-7)$ \\
\hline $\begin{array}{l}\text { Before treatment } \\
\text { sessions }\end{array}$ & & .41 & $\mathbf{. 0 0 8}$ & $<.001$ \\
\hline \begin{tabular}{l} 
At $\mathbf{4}^{\text {th }}$ week \\
\hline At 8 $^{\text {th }}$ week
\end{tabular} & & & $>.99$ & .072 \\
\hline
\end{tabular}




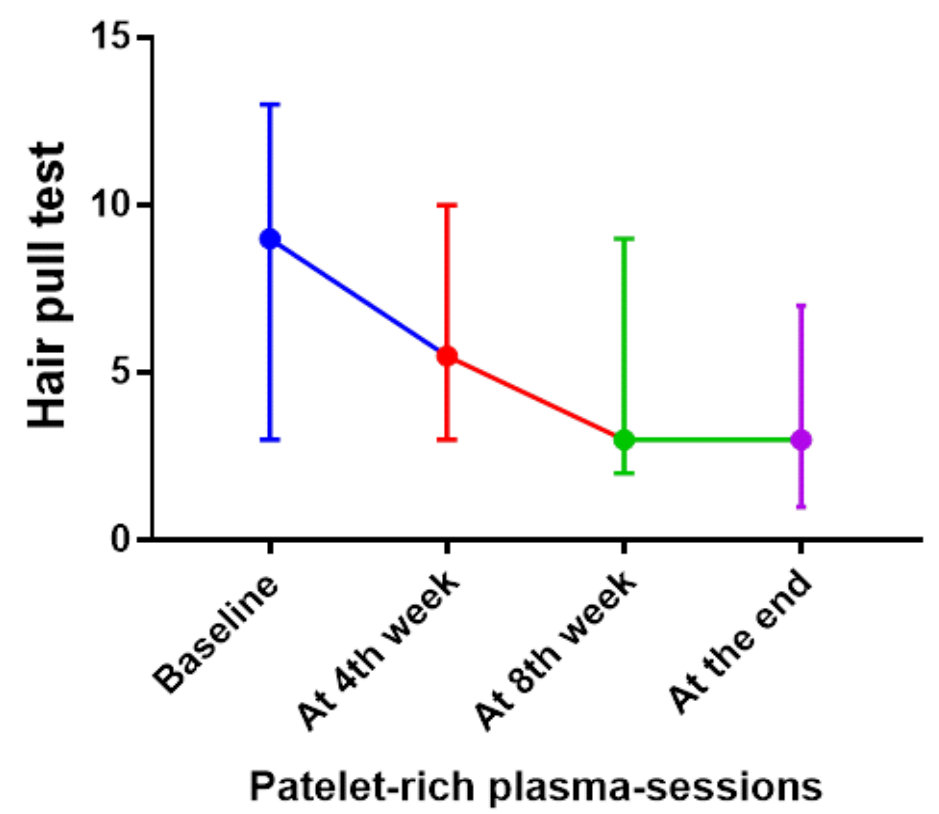

Fig (1): Error bars showing hair loss at the different time points during PRP injection . Data are median(range).
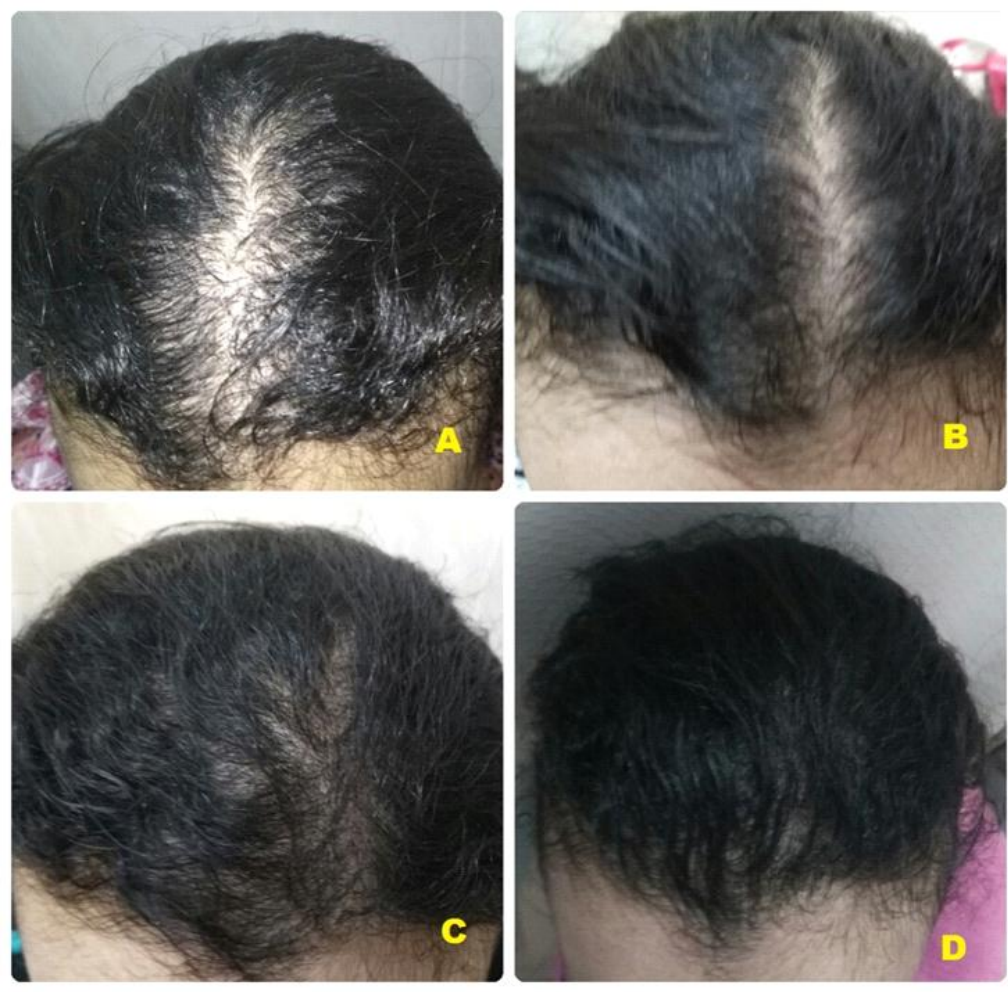

Fig (2): Female patient grade I AGA on Ludwing classification , (A) at start of therapy , (B, C) During treatment sessions and (D) After treatment with PRP injection sessions showing high improvement. 

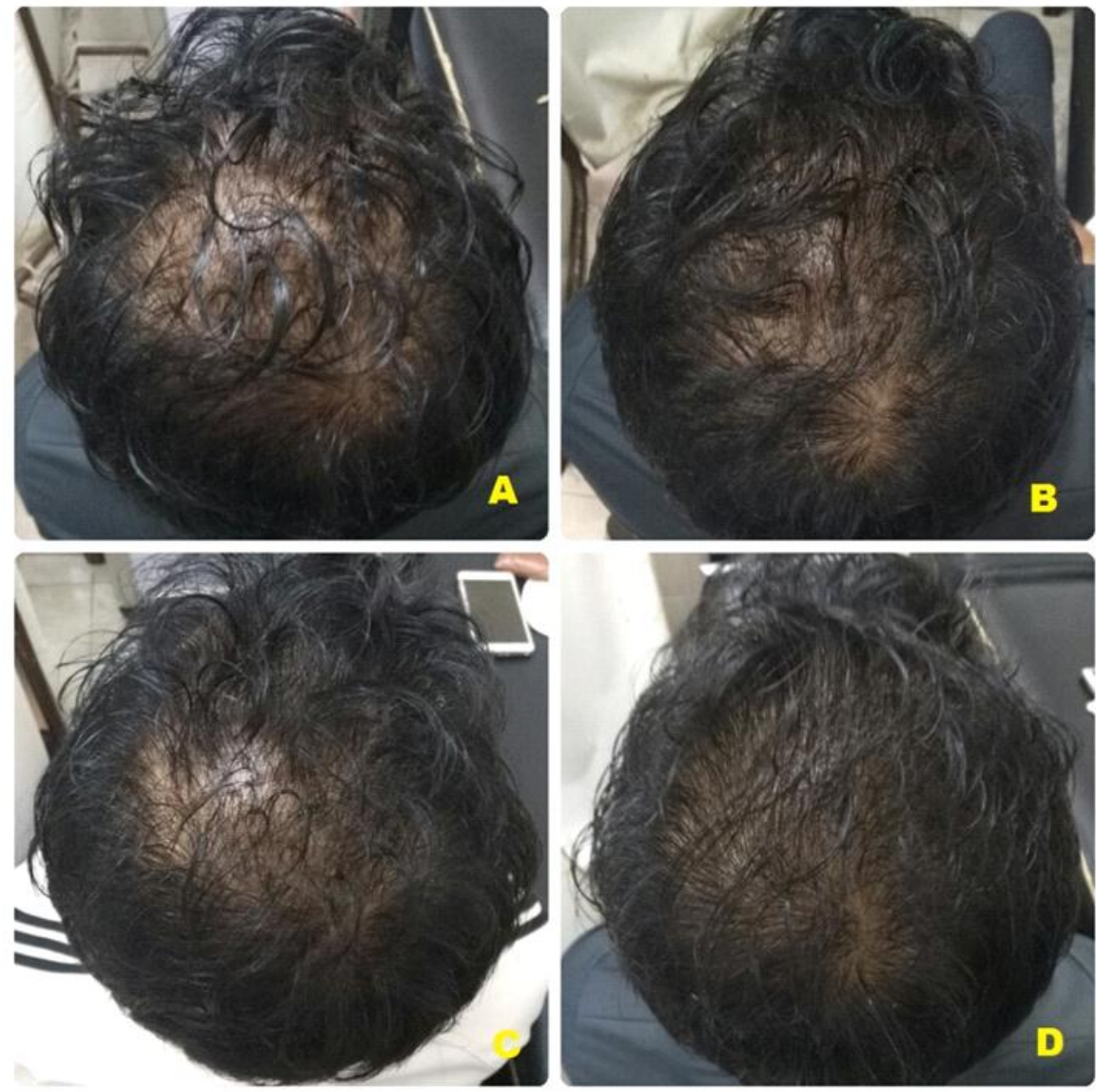

Fig (3): Male patient grade IIIv AGA on Hamilton-Norwood classification, (A) at start of therapy , $(B, C)$ During treatment sessions and (D) After treatment with PRP injection sessions showing moderate improvement.

\section{DISCUSSION}

Androgenetic alopecia (AGA) is a hereditary, androgen-dependent dermatological disorder .It is the most common non scarring alopecia that affects both males and females. The characteristic distribution of male pattern hair loss usually involves ; frontal, parietal, and central areas of the scalp, while female pattern hair loss presents with diffuse thinning of hair on the crown with preservation of the frontal hair line [6] .

The treatment of androgenetic alopecia should start as early as possible because untreated AGA progressively worsens . There are several treatment modalities available for promoting hair growth in AGA include; topical minoxidil, oral finasteride (type II 5- alpha reductase inhibitir) , ketoconazole, hormonal therapy, prostaglandin analogues, PRP, low-level laser therapy and hair transplant surgery.
Although currently; only topical minoxidil and oral finasteride are FDA approved [7].

PRP is an autologous preparation of human platelets in a small plasma volume, with a higher platelet concentration 4-7 times above the baseline. The growth factors and the bioactive molecules present in PRP promote; cell proliferation, migration, differentiation, and angiogenesis at the environment of administration , and also involved in the regulation of hair morphogenesis and hair cycle growth [8] .

Another action of PRP is the activation of extracellular signal-regulated kinase (ERK) and protein kinase $\mathrm{B}$ signaling that promotes cell survival and prevents apoptosis. PRP also increased the $\beta$-catenin activity and FGF-7 expression in dermal papilla cells . $\beta$-Catenin signaling is important in human follicle development and for the hair growth cycle [2] .

The present study revealed promising results about using platelet rich plasma in 
AGA. Regarding hair pull test ; before treatment, 8 patients $(80 \%)$ had a positive pull test with a mean number of 9.hairs. At 4 th and 8th week ,the hair loss decreased with a mean number of 5.5 and 3 hairs respectively. The hair loss was continued to decrease and all patient $(100 \%)$ had a negative pull test with a mean number of 2 hairs at the end of treatment this is highly statistically significant ( figure 1).

Our results were also evaluated by comparing the pre and post improvement photographs taken with the digital camera; by evaluating the final patient's satisfaction, physician's impressions and photographic assessment . The final patient's satisfaction was ; 8 patients (40\%) were satisfied and 12 patients $(60 \%)$ were not satisfied. The physician's impressions \& photographic assessment were ; 2 patients (10\%) as highly improved, 6 patients (30\%) as moderately improved, 6 patients $(30 \%)$ as mild improvement and 6 patients(30\%) as no change .

In the present work, no serious or persistent side effects were detected. Mild and transient adverse events were observed as follows: $60 \%$ experienced no side effects, $40 \%$ of patients experienced mild headache

Consistent with our results Betsi et al. [9] injected 42 patients with AGA by five sessions of PRP treatments in a period of two months. Before therapy, $90.5 \%$ of their patients had a +ve pull test with a mean number of 8 hairs. After the third session, the pull test was -ve in all patients with an average number of 3 hairs. Global images showed a significant improvement in the volume and quality of hair, which was confirmed by a high patient satisfaction .

Khatu et al . [10] in their study eleven (11) male patients with AGA treated with PRP sessions, and the treatment was repeated every 2 weeks, for a total of 4 times. Before therapy, all patients (100\%) had a +ve hair pull test with mean number of ten hair. After the fourth session, the pull test was -ve in 9 patients $(81.81 \%)$ with average number of 3 hairs. A significant reduction in hair loss was observed between first and fourth Injection and reported a moderate improvement in the volume and coverage of hair by global images

Our results were better than Borhan et al. [11] in their study which fourteen (14) cases of AGA in men injected with PRP, the cosmetic results and global pictures were disappointing, nevertheless, the patient self assessment showed a better hair texture in most cases. A few adverse effects such as; erythema, pain or headaches were observed.

Tawfik and Osman. [12] treated thirty female patients with AGA, with PRP injection sessions into a selected area, and with normal saline injection in another area as a placebo. The treatment repeated every week for a total of 4 sessions. Before therapy, all the patients $(100 \%)$ had a +ve hair pull test with a mean number of ten hairs in both PRP injected and placebo injected areas. At the 6month, the pull test was -ve in PRP injected areas in 25 patients (83\%) with average number of three hairs, global images showed a significant improvement in the volume and quality of hair, with a high patient satisfaction in PRP-injected areas. No major adverse effects observed during treatment only temporary pain and headache at the injection sites, and disappeared within a day.

\section{CONCLUSION}

Platelet rich plasma injection is a good option for treatment of AGA resulting in reduction of hair loss and promotion of new hair growth.

\section{REFERENCES}

1- Rallis E, Falidas E, Villias C. Amyopathic dermatomyositis-associated bilateral elbow ulcers successfully treated with autologous platelet-rich plasma. International journal of dermatology. 2014 Jan 1;53(1).

2- Li ZJ, Choi HI, Choi DK, Sohn KC, Im M, Seo YJ, et al . Autologous platelet-rich

plasma: a potential therapeutic tool for promoting hair growth. Dermatologic Surgery. 2012 Jul;38(7pt1):1040-6.

3-Starace M, Alessandrini A, D'Acunto C, Melandri D, Bruni F, Patrizi A, et al. Platelet-rich plasma on female androgenetic alopecia: Tested on 10 patients. Journal of cosmetic dermatology. 2019 Feb;18(1):59-64.

4-Kececi Y, Ozsu S, Bilgir O. A cost-effective method for obtaining standard platelet-rich plasma. Wounds. 2014;26(8):232-8. 
5-Eun HC, Kwon OS, Yeon JH, Shin HS, Kim BY, Ro BI, et al. Efficacy, safety, and tolerability of dutasteride $0.5 \mathrm{mg}$ once daily in male patients with male pattern hair loss: a randomized, double-blind, placebo-controlled, phase III study. Journal of the American Academy of Dermatology. 2010 Aug $1 ; 63(2): 252-8$.

6-Singhal P, Agarwal S, Dhot PS, Sayal SK. Efficacy of platelet-rich plasma in treatment of androgenic alopecia. Asian journal of transfusion science. $2015 \mathrm{Jul} ; 9(2): 159$.

7-Cervantes J, Perper M, Wong LL, Eber AE, Fricke AC, Wikramanayake TC, et al. Effectiveness of platelet-rich plasma for androgenetic alopecia: A review of the literature. Skin appendage disorders. 2018;4(1):1-1.

8-Marx RE. Platelet-rich plasma: evidence to support its use. Journal of oral and maxillofacial surgery. 2004 Apr 1;62(4):48996.
9-Betsi EE, Germain E, Kalbermatten DF, Tremp $\mathrm{M}$, Emmenegger V. Platelet-rich plasma injection is effective and safe for the treatment of alopecia. European Journal of Plastic Surgery. $2013 \mathrm{Jul}$ 1;36(7):407-12.

10-Khatu SS, More YE, Gokhale NR, Chavhan DC, Bendsure N. Platelet-rich plasma in androgenic alopecia: myth or an effective tool. Journal of cutaneous and aesthetic surgery. 2014 Apr;7(2):107.

11-Borhan R, Gasnier C, Reygagne P. Autologous platelet rich plasma as a treatment of male androgenetic alopecia: study of 14 cases. J Clin Exp Dermatol Res. 2015;6(04):1-6.

12-Tawfik AA, Osman MA. The effect of autologous activated platelet-rich plasma injection on female pattern hair loss: A randomized placebo-controlled study. Journal of cosmetic dermatology. 2018 Feb;17(1):4753.

shaban, S., Albalat, W., ElGarf, A. " The effect of Platelet Rich Plasma injection in How to cite 國 treatment of Androgenetic alopecia patients ". Zagazig University Medical Journal, 2020; (746-752): -. doi: 10.21608/zumj.2019.14509.1311 